\section{Las sesiones anatomoclínicas en México en los albores del siglo XXI}

\author{
Armando Garduño Espinosa, ${ }^{1}$ \\ Luis Heshiki Nakandakari ${ }^{2}$ \\ y Cecilia Ridaura Sanz ${ }^{3}$
}

1 México, Instituto Nacional de Pediatría, Departamento de Educación Médica Continua. Dirección postal: Instituto Nacional de Pediatría, Insurgentes Sur 3700-C, Col. Insurgentes Cuicuilco, México, D.F. CP 04530, México.

2 México, Instituto Nacional de Pediatría, Departamento de Pre y Posgrado.

3 México, Instituto Nacional de Pediatría, Servicio de Patología Posmórtem.
En muchos hospitales de todas partes del mundo se realizan periódicamente sesiones anatomoclínicas o clinicopatológicas, que constituyen un recurso fundamental en la enseñanza de los médicos de pre y posgrado y en la educación continua del personal de las instituciones de salud. Representan, a la vez, un indicador valioso de la calidad de la atención médica. Como ha señalado Lifshitz (1), la sesión anatomoclínica es la actividad académica que más se aproxima a un ejercicio de metodología diagnóstica sistematizada.

En el simposio sobre las autopsias y las leyes que organizó en 1970 en Nueva York la Foundation for the Advancement of Medical Knowledge, se concluyó que la sesión clinicopatológica era el mejor método de educación médica continuada (2). En este marco, educación médica continuada debe entenderse como el conjunto de actividades de enseñanza que desarrolla, mantiene, amplía, actualiza y profundiza los conocimientos, valores, actitudes y destrezas reconocidas por los profesionales médicos dentro de las disciplinas que sirven a los médicos para atender los problemas de salud de los pacientes y la comunidad (3). Cuando un médico compara sus diagnósticos nosológicos con los informes patológicos posmórtem, adquiere una buena idea de sus habilidades y de sus limitaciones como clínico. Esta función de la sesión anatomoclínica contribuye al aprendizaje ininterrumpido del médico y le permite mejorar constantemente su práctica profesional.

Las sesiones anatomoclínicas refuerzan el método clínico al proporcionar una oportunidad inigualada de revisar los conocimientos sobre una enfermedad y la forma de tratarla. Permiten proponer alternativas de diagnóstico y tratamiento, transformándose así en un mecanismo de primera línea para evaluar la calidad de la atención médica (1). Este proceso es una parte importante de la formación de los médicos residentes, por las ventajas que les ofrece discutir el caso de un paciente ante un auditorio. La participación de los médicos residentes en las sesiones anatomoclínicas facilita su evaluación no solo en cuanto a erudición profesional sino también a su actitud y habilidades para organizar, integrar, resumir, llegar a conclusiones y proponer decisiones.

Cabe destacar que las sesiones anatomoclínicas también favorecen la creación y adopción de nuevas normas y procedimientos acorde al avance de la medicina y fomentan la discusión interdisci- 
plinaria. Al mismo tiempo, promueven una actitud crítica y analítica, que se profundiza con el debate académico y el intercambio de puntos de vista diversos. Tienen, sin duda, una función relevante en la enseñanza de la medicina, ya que constituyen experiencias enriquecedoras y reflexivas. Por otra parte, entre sus objetivos figura el de generar nuevos enfoques por medio del cuestionamiento y la presentación de argumentos sólidos, lo que redunda en formalizar el método de tomar decisiones diagnósticas (1-4).

No obstante las aportaciones que brinda la práctica de las autopsias, hace ya algún tiempo que viene disminuyendo el interés tanto en obtenerlas como en realizarlas. En 1976, ya J. M. Prutting había hecho notar que el número de autopsias en los Estados Unidos de América había declinado claramente hasta corresponder a solo $20 \%$ de las defunciones (2). En Boston, ciudad que fue en una época el centro de sesiones de interés mundial, el porcentaje de autopsias se redujo de 75 a $38 \%$ de las defunciones registradas durante el período de 1960 a 1980. En relación con esas sesiones, que sirvieron de modelo para muchos otros hospitales y universidades, en el Hospital General de Massachusetts, el uso de los resultados de la autopsia como base para las sesiones anatomoclínicas disminuyó de $83 \%$ en 1965 a 14\% en 1980. Para 1983, en los Estados Unidos se efectuaban autopsias en solamente 15\% de las defunciones. Este fenómeno no ha resultado ser exclusivo de los hospitales de enseñanza estadounidenses, sino que se ha manifestado igualmente en los diversos países de Europa (5).

En lo que se refiere a México, en 1973 el porcentaje de autopsias en los hospitales del Distrito Federal fue 47\%; en 1994, 28\%; y en 1995, solo 15\% (6). Según informa Ángeles, durante 1985 el porcentaje de autopsias en el Instituto Nacional de la Nutrición disminuyó de $34,6 \%$ a $21,1 \%$ (5). En el Hospital del Niño, perteneciente a la anterior Institución Mexicana de Asistencia a la Niñez (IMAN), en 1975 se practicaban autopsias en 73,8\% de las defunciones, uno de los porcentajes más altos de los que se ha informado en el país (7). Ese mismo hospital, ahora conocido como Instituto Nacional de Pediatría, en 1988 registró autopsias para 37,1\% de las defunciones (8). Se calcula que en ese mismo año el promedio de autopsias realizadas en el resto del país fue de $13,5 \%$ (9).

Recientemente, el tema fue discutido en la Academia Nacional de Medicina con objeto de descubrir los factores que han determinado esa tendencia descendente. Se adujeron diferentes motivos; por ejemplo, que de cierto modo la muerte puede considerarse un fracaso de la medicina y con ella finaliza el estudio del paciente. Además, la muerte puede generar en el médico de cabecera un estado anímico adverso que le resta motivación para pedir la autorización de los familiares para una autopsia.

Por otra parte, hay quienes opinan que las tecnologías de diagnóstico están tan avanzadas, que cada vez se obtiene información más precisa en vida del paciente, lo que hace innecesaria la autopsia. Después de todo, esta representa un procedimiento costoso, en el que pueden gastarse hasta US\$ 1500 por caso $(7,10)$.

Hoy en día hay mucho temor a la crítica e incluso a las demandas medicolegales, cuando en estudios posmórtem se detectan enfermedades que no fueron diagnosticadas acertadamente en vida del paciente. Este quizá sea uno de los factores que más influye en la disminución de las autopsias $(7,9,10)$. Otras razones son que la autopsia tiene un lugar reducido en el ámbito privado $(6,8,11,12)$, que los clínicos minimizan su utilidad o la consideran innecesaria y que el interés de los patólogos se ha enfocado en otros campos como los de las patologías molecular, quirúrgica y experimental $(5,12,13)$.

\section{PANORAMA HISTÓRICO}

En México, las autopsias con fines didácticos datan de 1576 en el Hospital de Jesús (6). Antecedieron, por lo tanto, las enseñanzas de Thomas Sydenham (1624-1689), médico inglés a quien se atribuyen los fundamentos de la medicina clínica. Fue él quien estableció el estudio de los signos y síntomas mediante un método empírico y de observación, y el primer médico en hablar de las señales de la enfermedad - los síntomas (lo que el enfermo siente) y los signos (lo que el médico ve) - como clave del diagnóstico. Sydenham dedicó su inmensa labor a observar y clasificar las enfermedades, con el objetivo de descubrir en vida del enfermo las lesiones de los órganos internos. La clínica nació y se desarrolló sobre la relación entre lo observado en vida y lo hallado en el cadáver. Se fundó, pues, en lo que hoy llamamos correlación clinicoanatómica (6).

La primera gran obra que relacionó esos dos aspectos fue la del italiano Giovanni Battista Morgagni (1682-1771), De sedibus et causis morborum per anatomen indagatis [Del sitio y causa de las enfermedades por indagación anatómica], la cual está compuesta de una colección de historias clínicas con protocolos de necropsia y un comentario crítico. Publicada en 1761 en Venecia, la obra fue una aportación notable tanto a la anatomía patológica como a la clínica. En ella se describieron por primera vez múltiples enfermedades como la cirrosis hepática, los aneurismas sifilíticos, la tuberculosis renal y el absceso cerebral de origen ótico. Sin embargo, el desarrollo y perfeccionamiento de la clínica se debie- 
ron al esfuerzo de los médicos franceses en la segunda mitad del siglo XVIII y primera mitad del XIX, época en que la medicina francesa alcanzó su máximo esplendor (6). El método anatomoclínico nació con Marie-François-Xavier Bichat (17711802), quien en sus históricos trabajos señaló cómo la medicina ingresó al ámbito de la ciencia en el momento en que a la rigurosa observación del enfermo se agregó el examen de las alteraciones que presentaban sus órganos. La clasificación de enfermedades que propuso no se basa en los síntomas, sino en los resultados anatomopatológicos. Según Bichat, estos muestran la enfermedad y la tarea de los clínicos es indagar, mediante los signos y síntomas, las alteraciones anatómicas de los órganos internos. Nació así la doctrina anatomoclínica: estudiar los síntomas y signos de la enfermedad y ver después los cambios en los órganos para crear una semiología que permita predecirla (6).

Bichat señaló que el cuerpo expresa cada lesión por medio de síntomas. La evaluación de lo que el paciente siente y dice es esencial en la clínica, pero es indispensable que el médico tenga datos objetivos de la disfunción y que observe, palpe, percuta y escuche los órganos sanos para poder distinguirlos con claridad de los que están enfermos. De acuerdo con sus experiencias, "la tarea del clínico será reconocer en lo vivo con sus sentidos lo que revela la disección del cadáver". Estos preceptos han perdurado a través de los tiempos y han permitido a los médicos aprender el método anatomoclínico en el que la enseñanza y la investigación se hacen al pie de la cama del enfermo (6).

Las conferencias clinicopatológicas se instituyeron como actividad sistematizada en 1870 en la Escuela de Leyes de Harvard y se iniciaron en 1910 en el Hospital General de Massachusetts por influencia del médico norteamericano Richard Clarke Cabot. En 1923, los resúmenes de las sesiones empezaron a publicarse regularmente (14)

\section{LA SITUACIÓN ACTUAL}

El método clínico ha sido y continúa siendo la piedra angular del diagnóstico de las enfermedades con el apoyo de procedimientos paraclínicos de cada vez más complejidad tecnológica. Sin embargo, actualmente se subestima en la formación clínica del médico y, en cambio, se abusa de la tecnología, la cual, con sus espectaculares avances, amenaza con dejar atrás la enseñanza práctica de la medicina clínica. La integración de la clínica y los resultados de la autopsia han contribuido de manera sustancial a mejorar el conocimiento de las enfermedades, pero a pesar de ello, la concordancia entre el diagnóstico clínico y el posmórtem es variable. En México, esa concordancia es de $46,6 \%$ en un instituto nacional de salud; la cifra correspondiente al Instituto Karolinska en Suecia es de 57\%; en el hospital Peter Ben Brigham de Boston, (Massachusetts), Estados Unidos, es de 89\%; y en los hospitales del distrito South Lothian de Edimburgo, Reino Unido, de $42 \%(5,12)$. Estas cifras reafirman la importancia que tiene el estudio posmórtem para una mejor comprensión de los fenómenos clínicos, ya que se infiere que mientras más alta la concordancia clínicopatológica, mejor el procedimiento diagnóstico.

Para poder efectuar análisis más completos de casos, es indispensable continuar realizando autopsias. No se ha determinado cuál es el número ideal de autopsias, ya que ello dependería de cada hospital. Se ha sugerido de 30 a $50 \%$ de las defunciones hospitalarias, número que parece más bien arbitrario, ya que no se acompaña de ningún otro criterio. El porcentaje debe representar un número adecuado de casos para la enseñanza, la educación continua y la evaluación de la atención médica (15). Cabe reiterar que la autopsia ha sido y seguirá siendo una fuente inagotable de nuevos conocimientos, ya que por ese medio puede conocerse la naturaleza exacta de la mayor parte de las enfermedades. Además, la evaluación de los datos aportados por las autopsias en relación con los diagnósticos efectuados en vida del paciente revela si hubo errores de diagnóstico y por qué, con lo cual la autopsia se convierte en una fuente importante de retroinformación y en un indicador de la calidad del trabajo clínico $(5,12,15)$.

Entre los beneficios que se obtienen de las autopsias, se destacan los siguientes:

- Permiten ratificar o rectificar los diagnósticos clínicos, incluidos los que se obtienen mediante procedimientos de tecnología compleja como la tomografía computarizada, resonancia magnética, angiografía por sustracción digital y otras, y también estimar el valor de los medios terapéuticos empleados.

- Constituyen una forma de descubrir nuevas entidades patológicas.

- Son una fuente inextinguible de ideas para proyectos de investigación.

- Proporcionan estadísticas confiables sobre las enfermedades para la elaboración de informes.

- Contribuyen información útil para la orientación en genética.

- Representan un método excelente de enseñanza de pregrado y fomentan la educación continuada del personal médico y paramédico $(9,16)$.

- Favorecen la discusión y el intercambio interdisciplinarios de conocimientos. 
- Pueden ser un indicador de la calidad de la atención médica.

Por ser un extraordinario ejercicio de razonamiento y método de enseñanza en medicina, en cada sede debería presentarse por lo menos una vez al mes una sesión anatomoclínica en la que participen todos los médicos (17).

En una investigación llevada a cabo en los años ochenta por Ridaura et al., se estudiaron los factores que determinaron la autorización o denegación del permiso para la autopsia de un total de 469 defunciones en un hospital de tercer nivel de atención. Se hicieron autopsias de $274(58,4 \%)$ difuntos, las cuales correspondieron principalmente a hijos de madres analfabetas o con primaria incompleta, hijos de madres jóvenes y de madres solteras, y a hijos únicos. Más de la mitad de las madres con grados de escolaridad mediano y superior se negaron a autorizar la autopsia, mientras que los padres que más autorizaron el estudio posmórtem fueron obreros y campesinos. Los servicios que más autorizaciones recibieron $(>60 \%)$ fueron neonatología, cardiología e infectología, y los niños que menos autopsias tuvieron fueron los que murieron por leucemias y problemas quirúrgicos (18).

En otro hospital de tercer nivel de atención, las trabajadoras sociales registraron los siguientes motivos por los que no se habían realizado autopsias: en $32 \%$, los familiares no indicaron las razones para negar el permiso; en $8 \%$ se negaron por motivos religiosos y en $20 \%$ el permiso no se solicitó. Se concluyó que las causas principales de la disminución del número de autopsias habían sido la falta de interés del médico de cabecera y, quizá en menor grado, del patólogo, así como el desconocimiento de su utilidad (6). El interés en esas sesiones sigue declinando y si las hay, la asistencia es baja. Hay múltiples factores que se relacionan con este problema.

En primer lugar, en los hospitales hay servicios o departamentos que tienen sus propias sesiones clinicopatológicas que satisfacen sus necesidades y el personal ya no tiene necesidad de asistir a las sesiones generales. Los hospitales también ofrecen una variedad de sesiones didácticas: bibliográficas, radiológicas, clínicas, quirúrgicas, etc. Además, los grandes avances en informática han desplazado poco a poco a las sesiones hospitalarias como elementos fundamentales de actualización para los médicos.

Al mismo tiempo se ha reducido la antigua fascinación de asistir a discusiones académicas por grandes maestros. El tiempo de los médicos residentes está en gran parte ocupado por labores asistenciales, por lo cual evitan concurrir a las sesiones generales. Por último, cabe mencionar que falta el compromiso institucional (13).

\section{PROPUESTAS PARA MEJORAR LA SITUACIÓN}

Por todas las razones expuestas, y convencidos de los beneficios que aportan las sesiones anatomoclínicas en las diversas áreas del ejercicio de la medicina, hacemos las recomendaciones que siguen con el ánimo de despertar un nuevo interés en ellas.

- Concienciar a los médicos en cuanto a la importancia que tienen las autopsias y capacitarlos para obtener la autorización.

- Incluir en el planeamiento presupuestario institucional fondos para la práctica de estudios posmórtem y el mejoramiento constante de los métodos audiovisuales y de informática, de acuerdo con los avances tecnológicos de última hora.

- Idear estrategias para incrementar la motivación y el compromiso de los profesores en el desarrollo de estas actividades.

- Coordinar las labores asistenciales y educativas de los servicios o departamentos de una institución, de manera que las sesiones anatomoclínicas no interfieran con las actividades académicas generales.

- Como regla general para las sesiones, seleccionar casos de alta prevalencia y de interés común, que permitan mantener la actividad interdiciplinaria, sin menoscabo de los casos de baja incidencia o excepcionales.

- Establecer estándares e indicadores de calidad para evaluar las sesiones anatomoclínicas de forma que puedan modificarse cuando sea necesario mejorarlas.

- Poner en marcha un mecanismo para que las sesiones sean planeadas eficientemente por coordinadores de turno, de manera que tengan una dinámica fluida y cuenten con la participación ordenada de invitados y asistentes habituales, material de apoyo audiovisual de calidad, encuestas de evaluación y un horario regular.

- Establecer mecanismos para registrar las conclusiones y recomendaciones generadas en cada sesión, con el fin de darles seguimiento en las dependencias correspondientes y aplicarlas. Mediante este procedimiento se procura utilizar las sesiones como indicador de calidad con arreglo a la función asistencial, docente o de investigación de la institución.

- Sistematizar la publicación de resúmenes de las sesiones anatomoclínicas en la revista institucional.

Las sesiones anatomoclínicas generan un ambiente propicio para la reflexión, el intercambio académico por medio de la discusión y la confrontación de diferentes puntos de vista. Así el dogmatismo cede paso al surgimiento constante de argu- 
mentos y propuestas y se produce un espacio donde prevalecen la diversidad y la tolerancia (4).

Es por ello que este ambiente de alto nivel académico debe ser cuidado y cultivado. Las sesiones anatomoclínicas deben continuar cumpliendo con los objetivos de generar conocimientos, actitudes y valores sólidos y duraderos, y es importante que todo el personal de salud de las diversas instituciones contribuya en la medida de lo posible a su supervivencia y mejoría.

\section{SYNOPSIS}

\section{Postmortem clinical anatomy sessions in Mexico at the dawn of the 21st century}

Los autores presentan una apología de las sesiones anatomoclínicas basadas en los estudios posmórtem de casos, que contribuyeron enormemente al desarrollo de la medicina clínica pero hoy son de poco interés en el ámbito de los estudios de medicina. Sin embargo, las sesiones todavía constituyen un ejercicio de diagnóstico inigualado en el pregrado y un método excelente de educación continuada para los profesionales. Las autopsias permiten ratificar o rectificar los diagnósticos clínicos, incluidos los que se obtienen mediante procedimientos tecnológicos muy complejos; contribuyen al descubrimiento de nuevas entidades patológicas; estimulan los proyectos de investigación; proporcionan estadísticas confiables sobre morbilidad y mortalidad; revelan información útil en genética; favorecen la discusión y el intercambio interdisciplinarios de conocimientos y pueden servir como indicador de la calidad de la atención médica. Los autores recomiendan volver a cultivar el ambiente de alto nivel académico de las sesiones anatomoclínicas e instan a todo el personal de salud institucional a contribuir en la medida de lo posible a su supervivencia y mejoría.

\section{REFERENCIAS}

1. Lifshitz A, ed. La práctica de la medicina clínica en la era tecnológica. México, DF: Editorial UNAM; 1997. pp. 39-44.

2. Contreras-Rodríguez R. La autopsia. Gac Med Mex 1976;111:1-3.

3. Universidad Autónoma de México, Facultad de Medicina. Lineamientos para las actividades de educación médica continua. México, DF;1977. p. 7.

4. Viniegra VL. El camino de la crítica y la educación. Rev Invest Clin 1996;48: 139-158.

5. Ángeles A, Quintanilla ML, Muñoz FL, Espinoza VB, Victoria PP. Concordancia diagnóstica clinicopatológica en 429 autopsias del Instituto Nacional de la Nutrición Salvador Zubirán. Rev Invest Clin 1992;44:13-20.

6. Sánchez MJL, Uresca TC, Torre REF de la. La disección anatómica, las autopsias y su legislación en México durante los siglos XVI y XVII. Patología 1997; 35:13-21.

7. Flores-Barroeta F. Las prácticas de la autopsia en la enseñanza de la medicina. Gac Med Mex 1977;113:95-101.

8. Alonso P. Autopsias en los hospitales de enseñanza de México. Gac Med Mex 1988;124:349-352.

9. Benítez-Bribiesca L. La autopsia y la medicina moderna. Gac Med Mex 1988; 124:352-357.

10. Aguirre-García J. Panorama actual de las autopsias. Gac Med Mex 1988;124: 547-550.

11. Gutiérrez TG, Barbosa E, Acosta AE. Cómo mejorar las sesiones clínico-patológicas en pediatría. Rev Med IMSS 1981;19:141-143.

12. Estañol $\mathrm{B}$, ed. La invención del método anatomoclínico. México, DF: Editorial UNAM; 1996: p 329.
13. Albores-Saavedra J, Larraza O, OlveraRabiela J. La autopsia y su importancia en la educación médica continua. Gac Med Mex 1976;111:15-19.

14. Eddy MD, Clanton HC. The art of diagnosis, solving the clinicopathological exercise. N Engl J Med 1982;306: 1263-1268.

15. Ridaura SC. Recursos en la práctica de autopsias. Gac Med Mex 1997;133: 562-567.

16. Aguirre GJ. Panorama general de las autopsias. Gac Med Mex 1997;133: 547-550.

17. Aguirre GJ. La autopsia y la enseñanza de la medicina. Gac Med Mex 1997; 133:557-560.

18. Ridaura SC, López CE, García GI, Sosa MC. Factores de selección en la población autopsiada. Acta Pediatr Mex 1989; 10:149-153. 